\title{
Evaluating Lifting Barriers to Universal Access Project: A Step by Step Transformative Process for Increased Access to HIV Prevention and Treatment Services for Sexual Minorities in 2 Communities in Zimbabwe
}

\author{
Chibukire $\mathrm{N}^{*}$, Chingandu L and Ngwerume $\mathrm{P}$
}

SAfAIDS, 17 Beveridge Road, Avondale, Harare, Zimbabwe

${ }^{*}$ Corresponding author: Chibukire N, SAfAIDS, 17 Beveridge Road, Avondale, Harare, Zimbabwe, Email: ngoni@safaids.net

Citation: Chibukire N, Chingandu L, Ngwerume P (2016) Evaluating Lifting Barriers to Universal Access Project: A Step by Step Transformative Process for Increased Access to HIV Prevention and Treatment Services for Sexual Minorities in 2 Communities in Zimbabwe. J Aids Hiv Infec 2(2): 201. doi: 10.15744/2454499X.2.201

Received Date: September 20, 2016 Accepted Date: October 19, 2016 Published Date: October 20, 2016

\begin{abstract}
Background: In partnership with GALZ, Simbarashe network of PLHIV and ZWAAPV, SAfAIDS implemented Lifting Barriers to Universal Access Project: A Step by Step Transformative Process for Increased Access to HIV Prevention and Treatment Services for the Sexual Minorities in 2 Communities in Zimbabwe. The purpose was to increase access to HIV prevention, treatment, care and support services for key populations.

Methodology: Data was collected from the two intervention sites and one control site through focus group discussions with 12 key population representatives, 24 community leaders, and 26 community volunteers. In-depth key informant interviews were done with 24 service providers. Review of project reports and key documents was done.

Results: The project strengthened the capacity of implementing partners, traditional leaders and community volunteers to engage communities in addressing homophobia. GALZ capacity to work with non LGBTI communities was enhanced. Community leaders from Mhondoro-Ngezi were knowledgeable on the matter pointing out the need to embrace people irrespective of their sexuality diversity. There was improvement in knowledge levels in the communities on sexual and reproductive health rights. Through breaking the barrier of homophobia, the project realised increased access to HIV prevention, treatment and care services by general community members and LGBTI individuals.

Conclusions and Recommendations: It was learnt that it is possible to engage communities in discussions on sexual diversity. With appropriate skills and approaches, it is possible to conduct dialogues on sensitive topics in the communities. Advocacy for sexual rights of key population groups cannot be a stand-alone agenda, but should rather be embedded into aspect of HIV prevention strategies especially at community level. The project can be replicated and scaled up. There is need for assessment of other organizations whose core business is not LGBTI on their willingness to partake in advocacy for access to services by LGBTI individuals. Finally, there is need to have a protection plan for the champions and service providers who actively advocate for LGBTI issues in view of the hostile environment they operate in.
\end{abstract}

Keywords: HIV Prevention; Lifting Barriers; SAfAIDS

\section{Background information and project overview}

SAfAIDS in partnership with GALZ, Simbarashe Network of People Living with HIV (SNPLHIV) and Zimbabwe Women Against Poverty and Violence (ZWAPV), implemented the Lifting Barriers to Universal Access Project: A Step by Step Transformative Process for Increased Access to HIV Prevention and Treatment Services for the Sexual Minorities in 2 selected communities in Zimbabwe namely Mhondoro-Ngezi in Mashonaland West and Murehwa in Mashonaland East. The overall objective of this project was to increase access to HIV prevention, treatment, care and support services through innovative methodologies. Key activities involved development of well-targeted HIV and Gender Based Violence prevention toolkit for LGBTI communities, Information, Education and Communication (IEC) materials for LGBTI advocates and communities, strengthening community knowledge skills and competences on sex and sexuality as a strategy to reduce stigma and discrimination as well as conducting transformative community dialogues that helped in shaping discussions around policy, traditional and religious practices that create a barrier for LGBTI groups to access health services. Limited access to services is mainly due to social stigma and discrimination of sexual minorities by community members and health service providers $[1,2]$. Overall, the project sought to address the following challenges: 
- Weak advocacy efforts by activist groups in Zimbabwe, only one association (GALZ) that is advocating for the rights of gays and lesbians in Zimbabwe.

- The attitudes and perceptions of service providers towards sex workers and LGBTI groups have hindered their ability and willingness to provide HIV prevention, treatment and care services.

- Social stigma has kept epidemics among LGBTI groups hidden and unacknowledged.

- Fear of stigmatization and socio-cultural pressure often lead MSM to having multiple concurrent relationships with both men and women, increasing their risk for HIV infection.

- Heterosexist' health services that define only two sexes (men and women).

Achieving the $90 \times 90 \times 90$ global fast track by 2020 would not be possible if key populations are left behind. In addition, the 2030 goal of ending AIDS will be a mammoth task if HIV interventions do not meet the needs of key populations. The lifting barriers to universal access project were a SAfAIDS innovative approach to promote increased access to HIV prevention and treatment services. As part of evidence generation this 2-year project was evaluated in 2015. The purpose was to determine project effectiveness, relevance, and change or lack thereof at outcome and impact levels, generate key lessons learnt and identify promising practices for cross learning and replication purposes.

\section{Evaluation design and Methodology}

This study applied a cross-sectional assessment design, as events and situations were assessed at the same point in time using predominantly qualitative methods. Data was collected from the two (2) intervention sites (Mhondoro Ngezi and Murehwa) and one (1) control site (Seke Rural). During baseline, data was also collected from the same communities. A combination of qualitative and quantitative data collection techniques and tools within a participatory framework were employed for this evaluation. Within this framework, data was collected through separate focus group discussions with key population representatives, community leaders, and community volunteers. In-depth interviews with key informants representing service provider were also conducted.

Interviews of key informants, which were guided by a semi-structured interview guide, were held with 8 key informants ( 2 from intervention sites, 1 from the control site and 5 from GALZ-the implementing partner). In addition, 8 community leaders ( 4 from intervention sites and 4 from the control site) and 26 community volunteers ( 13 from intervention sites and 13 from the control site) took part in focus group discussions (FGD) which were guided by a checklist of key discussion themes $[3,4]$.

Review of project reports (workshop reports, annual progress reports) and other key documents was done for additional information to inform this evaluation.

\section{Key variables assessed were}

i. HIV prevention and SRHR knowledge among community leaders, CBVs, key populations and service providers

ii. Knowledge, attitudes and practices towards key population groups and sexual diversity among community leaders, CBVs, and service providers

iii. Factors influencing access to HIV, TB and sexual reproductive health services by general community and key populations iv. Capacity of $\mathrm{CBO}$ and community leaders to promote access to health services by key populations

\section{Key findings}

The project strengthened the capacity of implementing partners, traditional leaders and community volunteers to engage communities in addressing homophobia. This necessitated the trained leader and community volunteers to speak out against negative attitudes toward key populations. This resulted in key populations self-reporting that community were protecting, loving and respecting them (Figure 1).

In addition to the love, respect and protect finding above the following were the key results from the assessment:

\section{Project relevance and effectiveness}

- Service providers reported that the project helped them realize the need to respect people's sexual diversity and uphold their privacy as they come for services.

- Community leaders and Community Based Volunteers (CBVs) from intervention sites reported that the project enlightened them on the presence of LGBTI individuals in the community and the need to include them in SRHR and HIV programming.

- According to GALZ, the project helped in breaking new ground, enabling them to reach areas that were inaccessible before. GALZ had never implemented such interventions at community level to the general community members. In the past, they would target only LGBTI individuals. 


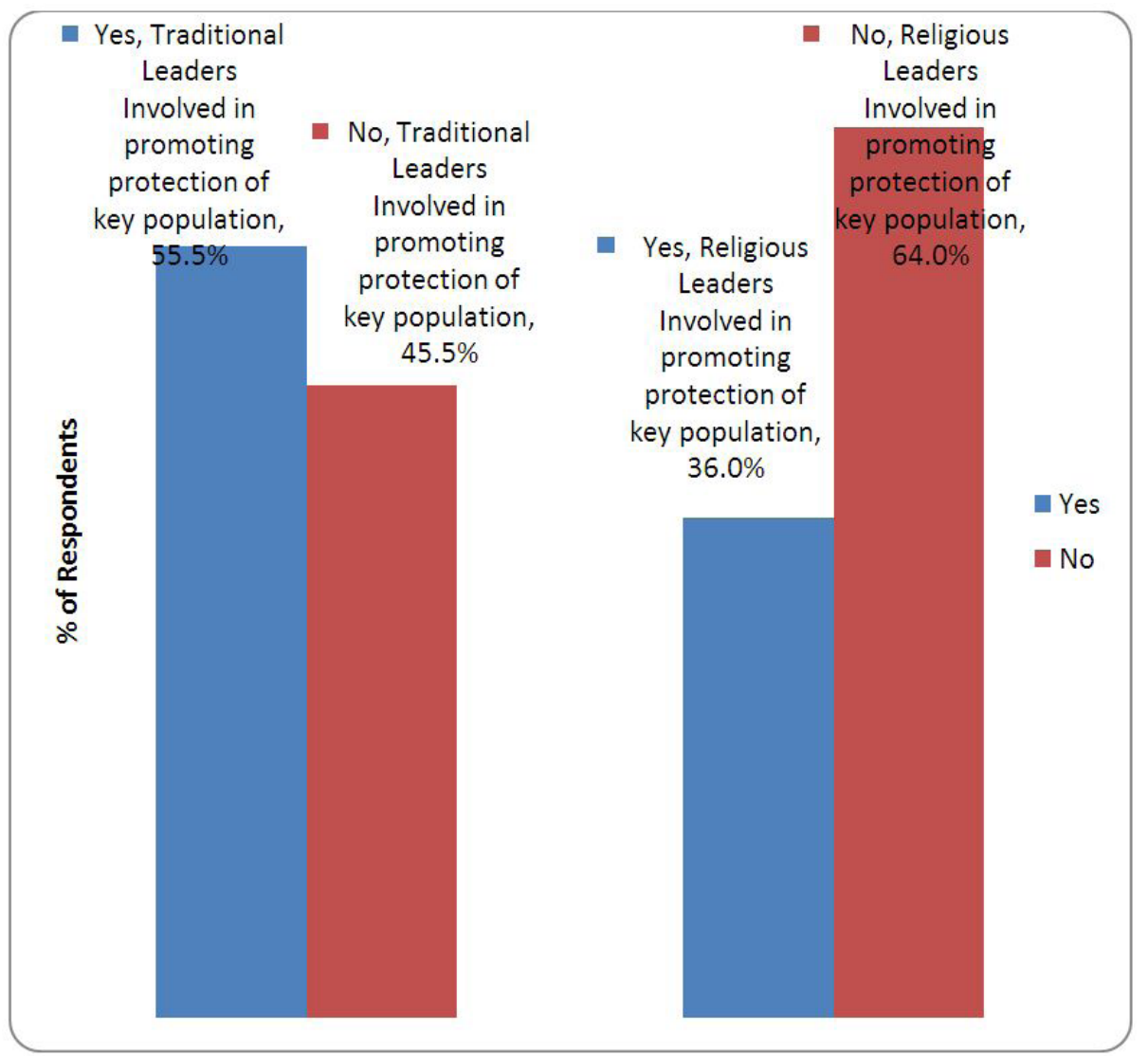

Figure 1: Proportion of Key Population Respondents Self-reporting Participation of Community Leaders in Protecting Them

\section{Knowledge, attitudes and actions towards sexual diversity}

- Community leaders from Mhondoro Ngezi (the intervention site) were knowledgeable on the matter pointing out the need to embrace people irrespective of their sexuality and sexual orientation. On the contrary, community leaders from Seke (control site) were not aware of what sexual diversity is and they linked it to evil forces which are not allowed in the community.

- CBVs from Mhondoro Ngezi, Murehwa and Seke were aware of sexual diversity and the need to accommodate people of diverse sexuality in programs that are being run in the community, especially those promoting access to health and SRHR.

- Service providers in Mhondoro Ngezi had a good understanding of sexual diversity and they underscored the need to avail services to all people without labelling them or discriminating them because of their sexual preferences.

\section{Knowledge and perceptions on Sexual and Reproductive Health and Rights (SRHR) for the general com- munity members and sexual minority groups}

- Knowledge of SRHR among community leaders was mainly high on the sexual aspect. However, most of the leaders were unable to identify reproductive health rights. The general perception with respect to SRHR amongst leaders from Mhondoro Ngezi was that LGBTIs have the same rights as other community members and they should be treated as such.

- CBVs from the two intervention sites were quite knowledgeable on SRHR issues, and were of the opinion that sexual minority groups have the same rights as any other member of the community. CBVs from Seke (control site) however do not feel that sexual minority groups have the same sexual and reproductive health and rights as other members of the community.

- Knowledge of SRHR was high amongst service providers. Service providers concurred that sexual minority groups deserve to be treated just like any other community member since they have the same SRH rights.

\section{Access to HIV prevention, treatment and care services by general community members and LGBTI indi- viduals}

- There has been a gradual improvement in terms of access to health services by general community members since the project was initiated.

- Community leaders, service providers and CBVs reported that the most beneficiaries reporting improved access to services are sex workers. 


\section{Roles of community leaders in promoting access to SRH and HIV services by general community mem- bers and sexual minority groups}

- Community leaders in Mhondoro Ngezi are actively involved in promoting access to services by members of the general community. They also act as champions promoting access to services for sex workers and to some extent LGBTI individuals.

- In Murehwa, community leaders who participated in the project are very active in promoting access to services by general community members even though there is still more work to be done for LGBTIs. Leaders who were not part of the project are not keen to be actively involved in promoting access to SRH and HIV services.

- In Seke (control site), community leaders are promoting access to SRH and HIV services by community members. However, they do not specifically focus on LGBTI individuals.

\section{Effectiveness of models employed to deliver the project}

- Despite the restrictive operational environment, the project was generally a success in the intervention sites. The project helped increase knowledge levels of community leaders, service providers and CBVs on the importance of including LGBTIs in SRHR and HIV programs conducted in the communities.

- According to GALZ, the project was a good entry point into communities which were regarded as hard to reach. The project created a good opportunity to engage community members, community leaders and service providers on this sensitive subject on sexuality and sexual orientation. This was possible through community dialogues where community members were able to freely discuss such issues. Using SRHR and HIV interventions as entry point to discuss sexuality issues also assisted in breaking the new ground.

\section{Key lessons learnt}

- It is possible to engage communities in discussions on sexual diversity, if right strategies and models are employed. Much as GALZ had been involved in advocacy for LGBTI people, there had never been efforts to reach out to non LGBTI people for support. The project showed that it can be done and there can be non LGBTI advocacy champions who can advocate for LGBTI issues in a hostile environment like in Zimbabwe. There was increased visibility of the LGBTI community due to skills and knowledge building approaches employed during programme implementation.

- The process was a learning curve for the LGBTI organizations which continue to use the IEC materials that were developed during the project. GALZ staff also learnt the skill of conducting dialogues for sensitive topics using SRHR and HIV interventions as an entry point. LGBTI people who attended the dialogues became more confident to be open about sexual diversity.

- There is acknowledgement of having improved on skill of planning for community activities i.e. being cognizant of safety and security issues of LGBTI people and champions. The other spin off of the project was that, GALZ started training journalists and other service providers after realizing that it is feasible to talk about sexual diversity with various groups.

- Advocacy for the sexual rights of key population groups like LGBTI communities cannot be a stand-alone agenda but rather should be integrated in HIV prevention strategies especially in rural communities where there are very few organizations advocating for the rights of key populations. Homophobia and transphobia have been politicized and so rights defenders felt intimidated to take up advocacy on the rights of LGBTI people.

- Because of same sex criminalization, the topic of sexual diversity is not adequately covered in trainings hence the service providers manifest negative attitudes out of ignorance because the attitudes of those that would have been sensitized, changed for the better.

\section{Key recommendations and conclusion}

Overall, data obtained from the project progress reports, community leaders, community volunteers, representatives of implementing partners, service providers, and representatives of key populations' shows that project had positive outcomes and impact in the intervention sites. There was increased knowledge and improved attitudes on sexual diversity and rights of key population groups among community leaders, community volunteers, $\mathrm{CBO}$ 's staff, and service providers in the intervention sites. In addition, the project strengthened the capacity of beneficiary CBOs on programming for key population groups.

In sites where the project was implemented, it was regarded a success especially by service providers since it provided them with a platform to break new ground and gave them an entry level into the community. This ensured that those people who were marginalised before could now be reached with information. In addition, the model helped in bringing to the fore perceptions of people in the community towards LGBTI individuals and in the process provided the forum for discussion about LGBTI issues in the community.

According to community leaders, the model helped in improving their knowledge levels on issues to do with LGBTIs. Now they realize the need to include these key populations in HIV programming. As a result of the way the project was conducted, focus around LGBTIs increased in the community. 
The same sentiments were shared by CBVs who confessed that the model employed to deliver the project has made their work in the community much lighter since they now know how to conduct themselves with different audiences. They now know how to bring issues of LGBTIs at public forums, as well as how to conduct dialogues on issues of SRH and HIV with respect to key populations.

Discussions with respondents during the end line evaluation revealed that the project interventions were relevant to the manifest and latent needs of the target populations as well as the situations being addressed. The program interventions which include capacity building of groups and CBOs involved in LGBTI interventions, production of IEC materials with messages on LGBTI issues and community dialogues were seen by the majority of stakeholders and beneficiaries as effective models to raise awareness on sexual diversity and SRH rights of LGBTI individuals. With regards to sustainability, the project strengthened CBOs and permanent community structures which are embedded in routine community activities.

Although the project was relevant, effective and resulted in achievement of intended outcomes, the legislative and political environment remained restrictive to programming for key populations to the extent that people cannot freely talk about sexual diversity issues. This is even more pronounced in rural areas (where the project was being implemented) compared to urban areas.

Based on the evaluation findings discussed, the following recommendations are presented:

- The Lifting Barriers to Universal Access Project should be replicated and scaled up. Since the community dialogues raise awareness among community members, there is need for more people knowing and talking about sexual diversity. Laws and policies on SRHR need to be unpacked.

- There is need for initiatives targeted at the key population groups so that they know more about themselves and their rights and are empowered enough to demand for services from various duty bearers.

- There is need for assessment, training and involvement of other organizations whose core business is not LGBTI. They need to be assessed on willingness to champion the cause then be trained on sexual diversity issues so that they also advocate for improved access to services for all key population groups - not just sex workers.

- In view of the hostile environment, there is need to have a protection plan for the champions and service providers who actively advocate for LGBTI issues. The champions and service providers need continued support and education on LGBTI issues so that they are properly nurtured. There has to be a plan on how to handle backlashes.

- Based on overall lessons, future phases need to target opinion leaders who influence individual beliefs and practices. The capacity building of religious leaders bore testimony to this as they pledged not to isolate LGBTI from their ministries and to model positive behaviour of, "Love, protection and respect for all."

\section{Acknowledgements}

SAfAIDS would like to acknowledge Aids Fonds for the financial support that enabled the implementation of the project and the undertaking of this evaluation. SAfAIDS also acknowledge the role played by Simbarashe AIDS Network, Seke Rural HomeBased Care, Gays and Lesbians of Zimbabwe (GALZ) and Zimbabwe Women against AIDS, poverty and violence (ZWAAPV) in sensitizing and mobilizing communities and stakeholders for this end line evaluation. In addition, these organizations participated as sources of data through key structured interviews. Without their support, undertaking this evaluation would have been difficult. Acknowledgement is also accorded to community leaders, LGBTI individuals, community volunteers, and general community members who were interviewed in this assessment.

A special thank you to the following SAfAIDS staff members for providing overall co-ordination and supervision of the study: Jabulani Mavudze (Head of Research Monitoring and Evaluation), Chido Musiya, and Leon-Say Mudadi. Technical guidance and review was provided by Lois Chingandu (SAfAIDS Executive Director) and Rouzeh Eghtessadi (SAfAIDS Deputy Director), and Ngoni Chibukire (Head of Programmes). Special acknowledgement to Caroline Maposhere who provided technical assistance in the evaluation process.

\section{References}

1. Lifting barriers for universal access to services final narrative report, SAfAIDS (2012).

2. SAfAIDS 5-year strategic plan (2010-2015).

3. ARASA Human rights violations reports.

4. GALZ annual reports (2010-2014). 


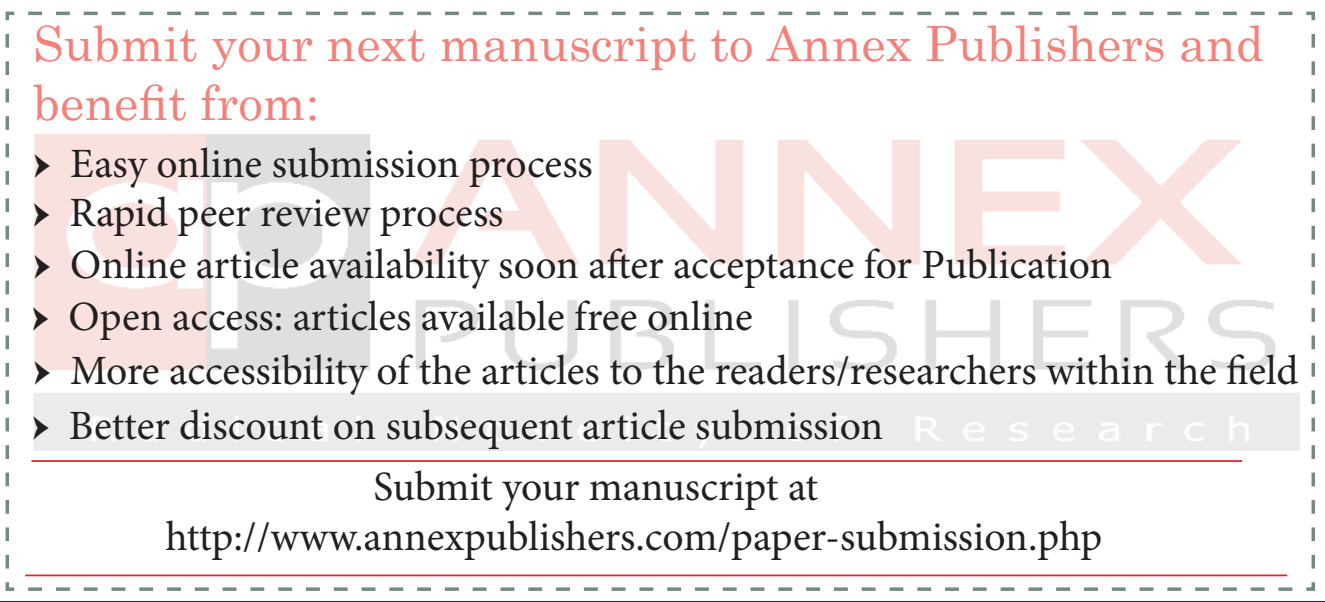

